\title{
Les laboratoires de dosimétrie passive
}

\author{
A. BIAU
}

Les laboratoires de dosimétrie passive peuvent se classer en trois catégories :

- les laboratoires de proximité qui traitent un nombre limité de dosimètres et sont implantés au sein des établissements dans lesquels travaillent les personnels surveillés ;

- les laboratoires généralistes traitent un grand nombre de dosimètres concernant des travailleurs exerçant dans des établissements multiples et avec des risques d'exposition très diversifiés ;

- les laboratoires aux statuts intermédiaires et particuliers.

Le laboratoire du CEA (chapitre 8) constitue un cas particulier dans la mesure où il a toujours été un laboratoire assurant le suivi dosimétrique réglementaire mais aussi un laboratoire de recherche faisant évoluer les techniques de dosimétrie.

\subsection{Les laboratoires de proximité}

Le laboratoire de la COGEMA/AREVA à La Hague est minutieusement décrit par Hubert Truffert dans le chapitre 11. L'autre laboratoire de COGEMA/AREVA est celui de l'Établissement de Marcoule dirigé par Michel Espagnan. Les aspects généraux sont du même type qu'à La Hague, les conditions de travail ne sont pas tout à fait identiques mais les études de poste permettent de bien adapter le mode de surveillance à la nature particulière des champs de rayonnements.

Dans cette catégorie de laboratoires de proximité, les autres laboratoires sont ceux du CNRS à Orsay et à Strasbourg. La problématique est la même que pour les laboratoires de COGEMA/AREVA avec un nombre limité de dosimètres et des personnels travaillant sur place. Les différences viennent du mode d'exposition car les sources ne sont pas exactement les mêmes. Les installations sont constituées d'accélérateurs de particules ou de réacteurs de recherche.

\subsection{Les laboratoires généralistes centralisés}

En dehors du laboratoire du Vésinet (SCPRI - OPRI- IRSN) faisant l'objet du chapitre 7, deux autres laboratoires ont couvert des milliers d'établissements et des 
dizaines de milliers de personnes réparties sur l'ensemble du territoire et ont été soumis aux mêmes contraintes de fonctionnement. Il s'agit du laboratoire Massiot et du Laboratoire central des industries électriques (LCIE).

Le laboratoire Massiot a été créé dans les années 1960 à Ailly-le-HautClocher (80) par Monsieur Massiot lui-même. Fabricant d'appareils de radiologie médicale et dentaire, il proposait un abonnement à ses clients lors de l'installation des appareils. Au fil du temps ce laboratoire est devenu « Massiot Philips», puis « Philips » avant d'être repris par le LCIE en 2005.

Les personnels suivis par ce laboratoire appartenaient au milieu médical. Ainsi en 2000, le laboratoire qui s'appelait alors Philips, assurait la surveillance dosimétrique de 23006 personnes. La technique dosimétrique utilisée a toujours été la dosimétrie photographique.

Le laboratoire du LCIE a été créé dans les années 1950 et concernait essentiellement le milieu industriel classique (non nucléaire) qui était en relation avec ce laboratoire pour des activités de contrôles traditionnels, notamment dans le domaine de la sécurité (pression, électricité, vapeur). Au cours des années, le domaine couvert par ce laboratoire, dirigé par Guy Le Roy et installé à Fontenayaux-Roses (92) s'est élargi à d'autres activités en médecine et dans le nucléaire civil.

En 2000, le nombre de personnes surveillées par le LCIE s'élevait à 42000 dont une majorité dans les champs d'activité de la radiographie et de la gammagraphie industrielle, environ 30 000, et les autres en milieu médical et nucléaire civil (agents des entreprises extérieures).

Jusqu'en 2000 la technique utilisée a été la dosimétrie photographique, papier puis film. En 2000, le LCIE a fait le choix de la technique OSL (voir chapitre 2) et s'est associé avec la société américaine Landauer et a pris le nom de LCIE Landauer puis Landauer Europe. Il a repris le laboratoire Philips et assure la surveillance dosimétrique des agents EDF soit environ 23000 personnes (voir chapitre 10).

\subsection{Les laboratoires « intermédiaires » - Cas particuliers d'EDF, du SPRA et du CERN}

EDF a mis en place en 1964 son propre laboratoire à Saint-Denis pour assurer la surveillance dosimétrique de ses propres agents avec un contrôle annuel du SCPRI selon les termes du décret d'avril 1975 (voir les chapitres précédents). Cette surveillance qui concernait environ 20000 agents EDF soumis essentiellement au risque d'exposition provenant des produits de fission $\left({ }^{131} \mathrm{I},{ }^{137} \mathrm{Cs}\right.$ notamment) et d'activation $\left({ }^{60} \mathrm{Co},{ }^{54} \mathrm{Mn}\right.$ et autres isotopes) était assurée par des dosimètres 
photographiques KODAK Type 2. À partir de 1998, cette prestation a été soustraitée au LCIE Landauer.

Le Service de protection radiologique des armées (SPRA) dispose d'un laboratoire de dosimétrie individuelle à l'origine situé à Arcueil puis à Clamart, qui suit les personnels de la Défense nationale travaillant dans les Installations nucléaires de base (INBS) et dans différents services utilisant des sources émettrices de rayonnements ionisants comme dans les services de santé aux armées (environ 8000 personnes). Ce laboratoire est plutôt de type généraliste car il est concerné par des sources de rayonnements différentes et les sites sont dispersés sur le territoire, mais il est quand même limité à moins de 20000 personnes surveillées (voir chapitre 9).

Enfin le Centre européen de recherche nucléaire (CERN) à Genève qui assure le suivi dosimétrique de tout le personnel intervenant dans ses installations transmettait au SCPRI puis l'OPRI les résultats concernant les agents français d'entreprises extérieures. En 2000 cela représentait 2700 personnes.

\subsection{Les contraintes de la distribution des dosimètres}

Il y a un aspect propre aux laboratoires centralisés. Cet aspect particulier n'est pas d'ordre technique ou scientifique, mais de façon plus terre à terre, concerne toutes les opérations de préparation et d'expédition des dosimètres ainsi que la réception des dosimètres et la mise à jour du fichier des abonnés.

Au début des années 2000, le laboratoire de l'OPRI expédiait chaque mois 120000 dosimètres à plus de 8000 établissements. Cette opération d'expédition est très lourde car les envois sont très hétérogènes dans des établissements pouvant disposer de deux dosimètres, comme les dentistes, ou 300 dosimètres comme les grands hôpitaux. Cela nécessite environ 60 hommes/jour et c'est la même chose pour la réception des dosimètres et leur préparation pour le traitement en laboratoire.

Les frais postaux sont considérables, c'est la raison pour laquelle pour diminuer le poids, seuls les films sont expédiés, la pochette et l'étui porte-écrans étant livrés lors du premier envoi, à charge pour l'utilisateur de mettre le film dans l'étui et la pochette.

Il existe un autre problème lié au cheminement postal aléatoire sur tout le territoire métropolitain, outre-mer et même à l'étranger (quelques pays africains). Lors de ces trajets, il est possible que les dosimètres soient exposés accidentellement, à proximité d'un colis radioactif ou d'un appareil à rayons X pour contrôler le courrier. 
Nous avions un établissement abonné situé dans une tour de la Défense dans laquelle tout le courrier était radiographié pour détecter des substances dangereuses. Cet abonné se rendait donc tous les mois au Vésinet pour chercher les dosimètres. Pour détecter toute anomalie, un dosimètre témoin était systématiquement joint aux dosimètres de chaque établissement abonné, ce dosimètre témoin servant également à déterminer un « voile de base », c'est-à-dire la densité optique d'un dosimètre non exposé aux rayonnements auxquels sont soumis les personnels professionnellement exposé de chaque établissement.

Enfin, une autre contrainte est la mise à jour du fichier général des abonnés du laboratoire, environ 2000 modifications, inscriptions et cessations chaque mois, ce qui correspond à un travail important, sans parler des litiges éventuels réglés par téléphone, fax ou courrier. Aujourd'hui, avec les moyens informatiques modernes, les abonnés peuvent directement effectuer les mises à jour. 